\title{
Efficient Distalization of Maxillary Molars with Temporary Anchorage Devices for the Treatment of Class II Malocclusion
}

\author{
Moschos A. Papadopoulos iD
}

Department of Orthodontics, Faculty of Dentistry, School of Health Sciences, Aristotle University of Thessaloniki, Thessaloniki, Greece

Cite this article as: Papadopoulos MA. Efficient Distalization of Maxillary Molars with Temporary Anchorage Devices for the Treatment of Class II Malocclusion. Turk J Orthod 2020; 33(3): 197-201.

\section{Main points:}

Orthodontic treatment of patients with Class II malocclusion by means of maxillary molar distalization can be very challenging.

Orthodontic miniscrew implants can be used as temporary anchorage devices (TADs) to enhance the anchorage and, if properly used, to counterbalance the side effects of conventional or noncompliant distalization approaches.

The TAD-supported amda ${ }^{\circledR}$ can be considered as a simple, noncompliant, minimally invasive, and very efficient approach that can be used for the comprehensive treatment of patients with Class II malocclusion.

\begin{abstract}
Treatment of Class II malocclusion often requires maxillary molar distalization. However, when applying distalization forces on the maxillary molars, anchorage loss may occur in different degrees not only during molar distalization (such as distal tipping of maxillary molars and mesial movement and proclination of the anterior teeth) but also during the subsequent stage of anterior teeth retraction (such as mesial movement of maxillary molars). All these movements are considered as unwanted side effects, which diminish the clinical effectiveness of distalization. Miniscrew implants can be used as temporary anchorage devices (TADs) to enhance anchorage and, if properly used, to counterbalance the side effects. Among the different available systems, the TAD-supported amda ${ }^{\circledR}$ can be considered as a simple, noncompliant, minimally invasive, and very efficient approach that can be used for the comprehensive treatment of patients with Class II malocclusion not only to distalize the maxillary molars bodily without or with minimal distal tipping and without proclination of the anterior teeth but also in combination with full-fixed appliances to retract and intrude the anterior teeth.
\end{abstract}

Keywords: Class II malocclusion, molar distalization, orthodontic anchorage techniques, orthodontic tooth movement, temporary anchorage devices

\section{INTRODUCTION}

When applying distalization forces on the maxillary molars, anchorage loss may occur in different degrees during not only molar distalization but the subsequent stage of anterior teeth retraction as well (1).

First, during maxillary molar distalization, anchorage loss can take place in the posterior or anterior area. Posterior anchorage loss includes distal tipping and/or distal rotation of the molars, which mainly depend on the biomechanics of the distalization system used (i.e., point of force application and force level and location of the center of resistance of the molars, which is considered to be at or very close to the trifurcation of their roots) (1$3)$. Anterior anchorage loss occurs as mesial movement and tipping of the canines and the first and/or second premolars, as well as proclination of the incisors and increase of the overjet.

Second, during the subsequent anterior teeth retraction that follows molar distalization, anchorage loss usually takes place in terms of mesial movement (and mesial tipping) of the maxillary molars because these teeth are used as anchor units at this stage of treatment (1).

Address for Correspondence: Moschos A. Papadopoulos, Department of Orthodontics, Faculty of Dentistry, School of Received: May 21, 2020 Health Sciences, Aristotle University of Thessaloniki, Thessaloniki, Greece 
For the moment, the only approaches that are not associated with anchorage loss during distalization involve the extraoral use of headgears or the intraoral use of skeletal or temporary anchorage devices (TADs) (4).

With regard to headgear treatment, it has to be noted that this approach requires maximum patient's cooperation, while it usually produces an orthopedic effect in terms of maxillary growth inhibition, which is not always desirable; therefore, most of the times, it cannot be utilized as part of our usual treatment plans.

In contrast, orthodontic TADs can be used for maxillary molar distalization, including all kinds of orthodontic (palatal) implants, orthodontic miniplates, or orthodontic miniscrew implants.

Previous attempts to distalize the maxillary molars using these measures with different distalization approaches have already been reported in the orthodontic literature; however, these are associated with various shortcomings.

For example, the modified Keles Slider has been used in conjunction with a single orthodontic implant positioned initially in the midpalatal suture of the palate and later in the paramedian area (5). Owing to the use of orthodontic implants instead of miniscrew implants, this approach is associated with more complicated and invasive procedures for the insertion and removal of the implant. Moreover, it needs more special, precise, and time-consuming laboratory work to connect the implant initially to the premolars (during molar distalization) and later to the molars (after molar distalization has been accomplished and during the stage of anterior teeth retraction).

Another example constitutes the use of the miniscrew-implant-supported distal jet, which is associated with the following problems: (a) a large acrylic button is still used, as with the conventional type of the appliance without miniscrews, which is usually related to hygiene problems and presence of inflammation of the underlying soft tissues; (b) the TADs are inserted between the roots of the first and second premolars or between the second premolars and first molars, a fact that is associated with a risk of root injuries of these teeth not only while inserting the implants but also during drifting of the premolars distally; and (c) to use the same appliance for the subsequent anterior teeth retraction, the TADs have to be removed and new TADs have to be inserted in different locations to facilitate the further use of the distal jet for this purpose. This procedure is time consuming and is associated with additional stress and costs for the patient (6).

Furthermore, the Beneslider (7), a distalization system also supported by miniscrew implant anchorage, presents the shortcoming that two TADs are inserted in the midpalatal suture of the palate. However, it is already known that there is still connective tissue in the midpalatal suture even in the adults and that the suture is not following a straight line $(8,9)$. Thus, the contact surface between the thread of the TADs and the bone is significantly decreased. Because the anchorage and thus the stability of the miniscrew implants succeed only because of mechanical retention and not through osseointegration, the decreased bone-toimplant interface may lead to a significant increase of the risk of mobility or even to the failure of the TADs. Furthermore, since both TADs are inserted in the midpalatal suture, the distal TAD is inserted in an area with decreased bone height; apart from the significant increase in the risk for immobility, most likely, it perforates or penetrates the lower border of the sinus. Finally, the length of the two Benetubes (that connect the palatal arch wire with the molar bands and transfer the distalization force to the molars) cannot be individually adjusted. Therefore, when distalizing maxillary molars with large roots, the line of action of the force system may lie more occlusally to their center of rotation, and this can lead to distal tipping of these teeth during distalization.

Another noncompliant distalization appliance that has been supported with miniscrew implant anchorage is the "bone-anchored Pendulum appliance" (10). This appliance also presents some significant problems and shortcomings: (a) similar to the TAD-supported distal jet, a large acrylic button is also still used as with the conventional type of the appliance without TADs, which usually leads to hygiene problems and inflammation of the underlying soft tissues, which in turn, may cause mobility and failure of the TADs; (b) the point of force application is located on the crown level of the maxillary molars; thus, distal tipping in different degrees of the molars usually takes place; $(c)$ the maxillary molars are moving on a "pendulum arc" while distalizing; therefore, distal rotation of the molars always occurs, while the maxillary arch is usually significantly constricted posteriorly; (d) there is no auxiliary or any stop to prevent the movement of distalization of the molars; hence, the molars may be significantly overcorrected; in some cases, if the patient misses some appointments for any reason, the molars may end up close to the midpalatal suture.

Finally, orthodontic miniplates or miniscrew implants (that are positioned in the posterior area) can be used in conjunction with fixed appliances (braces) to distalize the maxillary molars. This is usually done in an indirect manner, i.e., by means of open-coil springs that are compressed between the maxillary molars and premolars or canines, while the premolars or canines are connected to the TADs. In these cases, because the point of force application and the level of the distalization force always lie on the crown level (i.e., below the center of resistance of the maxillary molars), distal tipping (posterior anchorage loss) always takes place.

The author of this article has recently developed a device, the miniscrew-implant-supported advanced molar distalization appliance (amda ${ }^{\oplus}$; Dentaurum, Ispringen, Germany) (Figures 1 and 2 ), which constitutes a novel technique that eliminates the aforementioned drawbacks and side effects of the conventional and noncompliant distalization appliances, i.e., the anchorage loss of the anterior dental unit (in terms of mesial movement of the premolars and canines and proclination of the incisors) and of the posterior dental unit (in terms of distal tipping and rotation of the molars) during maxillary molar distalization (Figure 3a), as 
well as the anchorage loss of the posterior dental unit (in terms of mesial movement of the molars that have been previously distalized) during anterior teeth retraction (Figure $3 \mathrm{~b}$ ). During molar distalization with amda', the molars are distalized almost bodily, while the appliance remains invisible $(11,12)$. Thereafter, the same appliance after a small chairside intraoral modification can provide the necessary anchorage for the subsequent anteri-
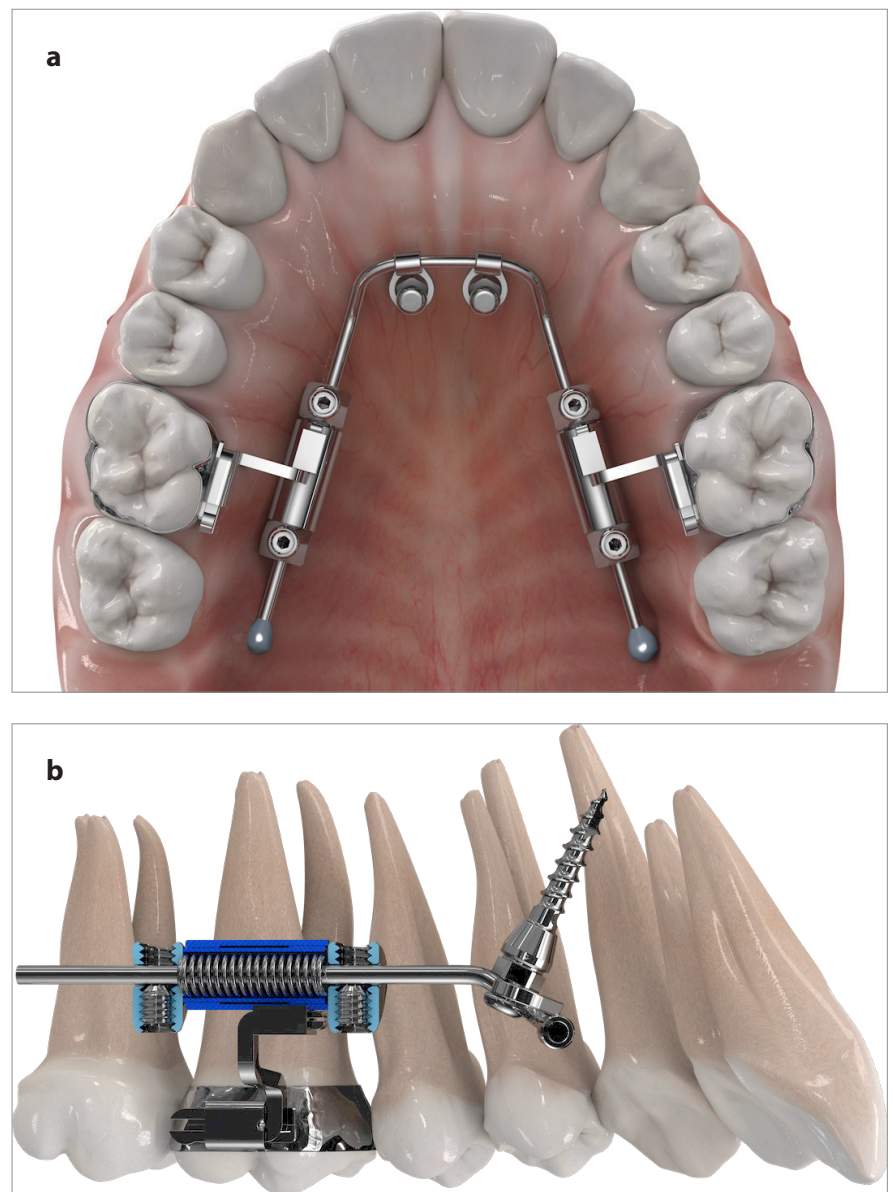

Figure 1. a, b. Three-dimensional virtual representation of the amda $^{\oplus}$ (a) Occlusal view, (b) Sagittal view

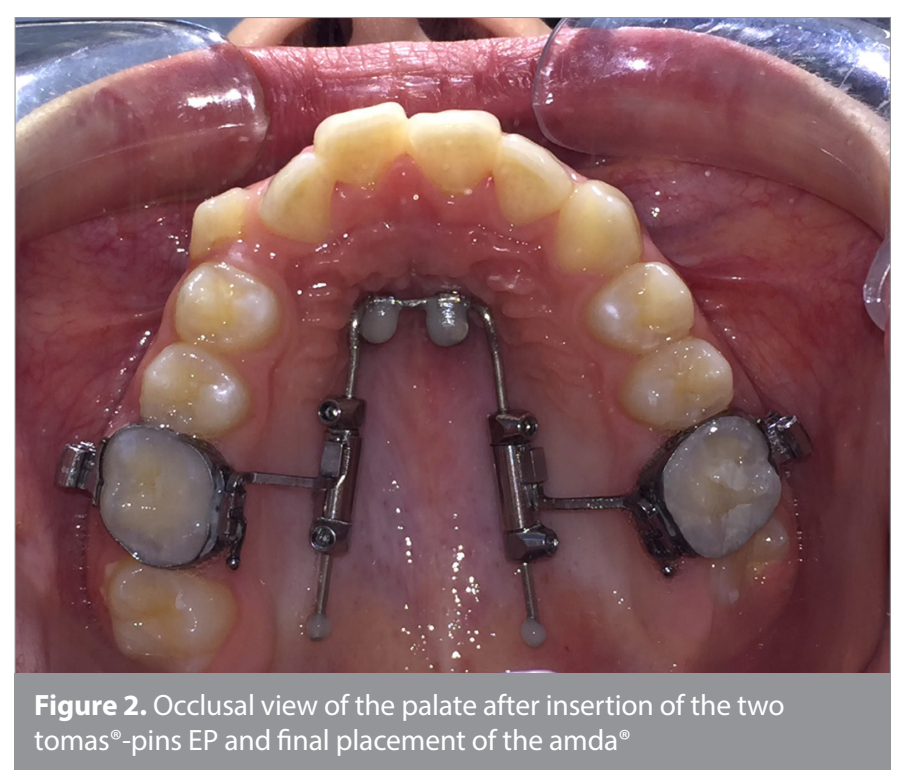

or teeth retraction, which is performed in conjunction with the conventional fixed appliances.

The amda ${ }^{\circledR}$ consists of an active and an anchorage unit. The active unit bilaterally uses an apically positioned wire-tubing system, including the active element of amda $^{\circledR}$, i.e., compressed nickel-titanium open-coil springs, which provide the necessary force for molar distalization (Figure 1b).

The anchorage unit consists of two self-drilling miniscrew implants, the tomas ${ }^{\oplus}$-pins EP (Dentaurum, Ispringen, Germany), with which the skeletal anchorage of amda $^{\circledR}$ is fulfilled. Two TADs are always used to skeletally anchor the amda $^{\circledR}$ to (a) avoid possible rotational movements of the appliance in case of asymmetrical force application (e.g., for bilateral molar distalization of different amount between right and left sides or unilateral distalization) and (b) enhance the stability of the appliance. The TADs are inserted paramedian in the anterior region of the palate, especially 6-9 $\mathrm{mm}$ posterior to the incisive foramen and 3-6 $\mathrm{mm}$ paramedian because it was found that this site offers the highest amount of bone support (Figures 1 and 2) (13).

Further details regarding the clinical application of amda ${ }^{\circledR}$ have been described in the literature $(4,14)$. Initial treatment results highly support this new approach since the TAD-supported amda $^{\circledR}$ can be used efficiently to not only distalize the maxillary molars bodily but also subsequently retract the anterior teeth without relying on patients' cooperation, in other words, for the comprehensive management of Class II malocclusion (Figure 4).

a

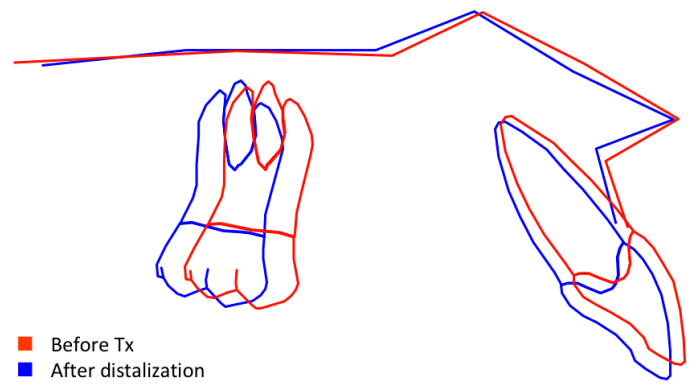

b

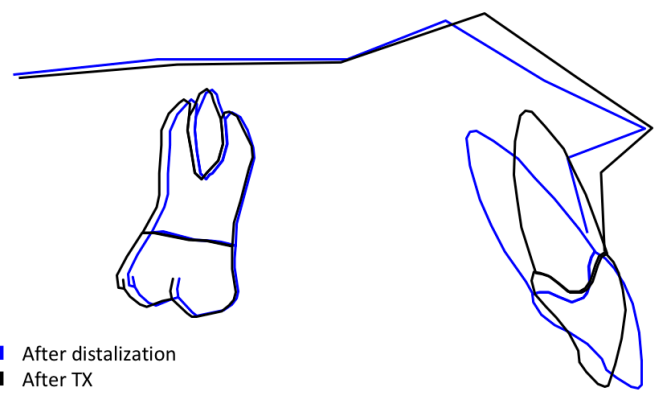

Figure 3. a, b. Superimpositions of the lateral cephalometric tracings on the maxillary plane of a patient with Class II malocclusion treated with the amda ${ }^{\oplus}$ : (a) Before and after distalization of the maxillary molars; (b) After distalization of the maxillary molars and after retraction of the anterior teeth 

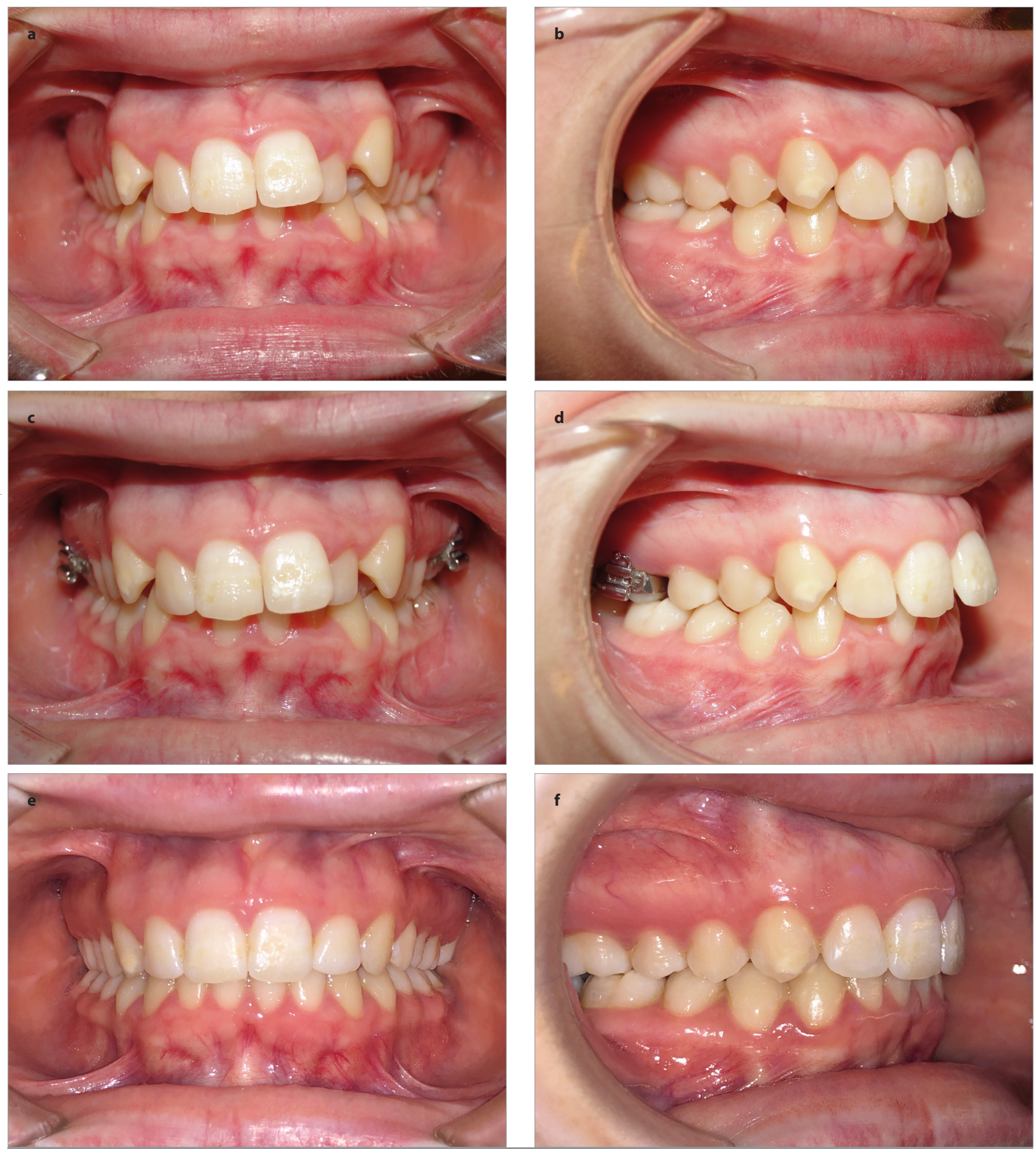

Figure 4. a-f. Intraoral photographs of a 13-year-old female with Class II malocclusion Treated with the amda ${ }^{\oplus}$ ( $(a, b)$ Before initiation of treatment; $(c, d)$ Immediately after completion of distalization of the maxillary molars with the amda ; $(e, f)$ Immediately after completion of treatment and after debonding of the maxillary and mandibular dental arch

As mentioned above, the amda ${ }^{\oplus}$ uses two TADs as stationary anchorage to resist the mesial-directed reciprocal forces produced by the coil springs during molar distalization, as well as later to support the subsequent anterior teeth retraction. This way, the side effects of anchorage loss of the anterior dental unit during molar distalization and that of the posterior dental unit during the subsequent anterior teeth retraction are eliminated or at least substantially minimized.

In addition, the point of force application exerted by the palatally positioned nickel-titanium open-coil springs encased in the tubing system of the amda $^{\circledR}$ telescopes passes almost through 
or very close to the center of resistance of the maxillary molars (at the roots' trifurcation). Thus, an almost pure bodily molar distal movement is produced, while a distal molar crown tipping is avoided.

Furthermore, because the molars are forced to slide on the am$\mathrm{da}^{\circledR}$-palatal arch and are guided through the amda ${ }^{\circledR}$ telescopes, which are all palatally positioned and run parallel to the maxillary occlusal plane, no or minimal rotation of these teeth is usually observed during distalization. However, if any molar rotation occurs, the amda ${ }^{\circledR}$ connectors can be bend with antirotation accordingly during the course of treatment to counteract this side effect.

Finally, during the subsequent stage of the retraction of the anterior teeth, posterior anchorage of the first maxillary molars is efficiently supported by the same appliance after a small and easy chairside intraoral adjustment of the amda ${ }^{\circledR}$ telescopes; thus, no mesial movement of the maxillary molars that have been just distalized is observed.

The amda ${ }^{\circledR}$ can also be very efficiently applied unilaterally in Class II subdivision cases requiring unilateral distalization only of one maxillary molar in one side or in asymmetrical Class II cases where asymmetrical distalization of the maxillary molars between right and left sides is indicated.

Although the amda $^{\oplus}$ has been primarily developed for distalization of the maxillary first molars, this approach may also be utilized efficiently for the bilateral or unilateral mesialization of the maxillary molars or to distalize the first molar on one side of the maxilla and mesialize the first molar on the contralateral side.

\section{CONCLUSION}

Among the different available systems, the TAD-supported amda ${ }^{\circledR}$ can be considered as a simple, noncompliant, minimally invasive, and very efficient approach that can be used for the comprehensive treatment of patients with Class II malocclusion not only to distalize the maxillary molars bodily without or with minimal distal tipping and without proclination of the anterior teeth but also in combination with full-fixed appliances to retract and intrude the anterior teeth.

Peer-review: Externally peer-reviewed.

Author Contributions: Supervision - M.A.P.; Design - M.A.P.; Supervision - M.A.P.; Resources - M.A.P.; Materials - M.A.P.; Data Collection and/ or Processing - M.A.P.; Analysis and/or Interpretation - M.A.P.; Literature Search - M.A.P.; Writing Manuscript - M.A.P.; Critical Review - M.A.P.

Conflict of Interest: The author owns a US and a German patent of the amda ${ }^{\oplus}$.
Financial Disclosure: The authors declared that this study has received no financial support.

\section{REFERENCES}

1. Papadopoulos MA, editor. Orthodontic treatment for the Class II non-compliant patient: Current principles and techniques. Elsevier, Mosby, Edinburgh, 2006.

2. Papadopoulos MA, Mavropoulos A, Karamouzos A. Cephalometric changes following simultaneous first and second maxillary molar distalization using a non-compliance intraoral appliance. J Orofac Orthop 2004; 65:123-36. [Crossref]

3. Mavropoulos A, Karamouzos A, Kiliaridis S, Papadopoulos MA. Efficiency of non-compliance simultaneous first and second upper molar distalization: A 3D tooth movement analysis. Angle Orthod 2005; 75: 532-9.

4. Papadopoulos MA, Tarawneh F. The use of miniscrew implants for temporary skeletal anchorage in orthodontics: a comprehensive review. Oral Surg Oral Med Oral Pathol Oral Radiol Endod 2007; 103: e6-15. [Crossref]

5. Keles A, Erverdi N, Sezen S. Bodily distalization of molars with absolute anchorage. Angle Orthod 2003; 73: 471-82.

6. Bowman SJ. The evolution of the Horseshoe Jet. In: Papadopoulos MA, editor. Skeletal anchorage in orthodontic treatment of Class II malocclusion: Contemporary applications of orthodontic implants, mini-screw implants and mini plates. Edinburgh: Elsevier, Mosby, 2015; 168-70. [Crossref]

7. Wilmes B. The Beneslider and Pendulum B appliances. In: Papadopoulos MA, editor. Skeletal anchorage in orthodontic treatment of Class II malocclusion: Contemporary applications of orthodontic implants, mini-screw implants and mini plates. Edinburgh: Elsevier, Mosby, 2015; 174: 1-7. [Crossref]

8. Melsen B. A histologic study of the influence of sutural morphology and skeletal maturation on rapid palatal expansion in children. Tram Eur Orthod Sot 1972; 499-507.

9. Melsen B. Palatal growth study on human autopsy material: A histologic microradiographic study. Am J Orthod 1975; 68: 42-54. [Crossref]

10. Kircelli HB, Pektas ZO. The bone-anchored Pendulum appliance. In: Papadopoulos MA, editor. Skeletal anchorage in orthodontic treatment of Class II malocclusion: Contemporary applications of orthodontic implants, mini-screw implants and mini plates. Edinburgh: Elsevier, Mosby, 2015: 186-8. [Crossref]

11. Papadopoulos MA, editor. Skeletal anchorage in orthodontic treatment of Class II malocclusion: Contemporary applications of orthodontic implants, mini-screw implants and mini plates. Edinburgh: Elsevier, Mosby, 2015. [Crossref]

12. Papadopoulos MA. The advanced molar distalization applicance: $A$ novel approach to corect Class II Malocclusion. Recent Patents on Biomedical Engineering 2010; 3: 6-15. [Crossref]

13. Bernhart T, Vollgruber A, Gahleitner A, Dörtbudak O, Haas R. Alternative to the median region of the palate for placement of an orthodontic implant. Clin Oral Implants Res 2000; 11: 595-601. [Crossref]

14. Papadopoulos MA. Effective treatment of Class II malocclusion with the mini-screw-implant supported amda@. In: Park JH, editor. Temporary Anchorage Devices in Clinical Orthodontics. Hoboken, NJ: Wiley-Blackwell 2020; 153-60. [Crossref] 